\title{
Adequação nutricional e desperdício de alimentos em Centros de Educação Infantil
}

\author{
Nutritional adequacy and food waste \\ in Early Childhood Education Centers
}

\author{
Camila Alves Nogueira de Souza ${ }^{1}$ \\ Giovana Longo-Silva ${ }^{1}$ \\ Risia Cristina Egito Menezes ${ }^{1}$ \\ Alyne da Costa Araujo ${ }^{1}$ \\ Maysa Helena de Aguiar Toloni ${ }^{2}$ \\ Maria Alice de Araújo Oliveira ${ }^{1}$
}

${ }^{1}$ Faculdade de Nutrição, Universidade Federal de

Alagoas. BR 104 Norte Km 97, Tabuleiro do Martins.

57072-900 Maceió AL

Brasil. camilaa.nogueira@

hotmail.com

${ }^{2}$ Departamento de Ciência

dos Alimentos, Universidade

Federal de Lavras. Lavras

MG Brasil.

\begin{abstract}
Analyzing quantitative and qualitative aspects of school meals represents the initial stage to ensure that the National School Food Program (PNAE) is an effective space for the promotion of healthy eating habits and establishing individual rights. The objective of this study was to evaluate the nutritional adequacy of meals served and consumed, as well as quantifying food waste in Child Daycare Centers in Maceió, in the state of Alagoas. All meals served and consumed by 359 children (aged 17 to 63 months) were analyzed by the direct weighing method for three non-consecutive days in all institutions. Food waste was determined by the leftover factor and the nutritional value of meals served and consumed was compared to the PNAE reference values regarding energy, carbohydrates, protein, lipids, vitamins $A$ and $C$, iron, calcium, zinc, magnesium and fiber. Of the 85 meals/preparations featured on the menu, 58 (68\%) revealed a high leftover percentage; in excess of $10 \%$. The meals served and consumed met approximately 50\% of the recommended energy and macronutrient values. The difference between the averages of the nutrients offered and consumed was statistically significant $(p<0.05)$ for fiber, vitamin $A$, vitamin $C$, calcium, iron and zinc.
\end{abstract}

Key words Children, Daycare centers, School food, Nutritional recommendations, Food wastage
Resumo Analisar aspectos quantitativos e qualitativos da alimentação escolar constitui-se de etapa primordial para fazer do Programa Nacional de Alimentação Escolar (PNAE) um espaço efetivo para a promoção da alimentação saudável e formação de sujeitos de direitos. O objetivo deste estudo foi avaliar a adequação nutricional das refeições servidas e consumidas e quantificar o desperdício de alimentos em Centros de Educação Infantil de Maceió/AL. Foram analisadas por três dias não consecutivos, pelo método de pesagem direta, as refeições de 359 crianças (17 e 63 meses). $O$ desperdício de alimentos foi determinado pelo indice de resto-ingestão e o valor nutricional das refeições servidas e consumidas foram comparadas com os valores de referência do PNAE para energia, carboidrato, proteína, lipídio, vitaminas A e C, ferro, cálcio, zinco, magnésio e fibra. Dos 85 alimentos/preparações presentes no cardápio, 58 (68\%) apresentaram percentual de resto-ingestão, superior a $10 \%$. As refeições servidas e consumidas atenderam aproximadamente $50 \%$ do recomendado para energia e macronutrientes. A diferença entre as médias dos nutrientes ofertados e consumidos foi estatisticamente significante ( $p<$ $0,05)$ para fibra, vitamina A, vitamina $C$, cálcio, ferro e zinco.

Palavras-chave Crianças, Creche, Alimentação escolar, Recomendações nutricionais, Desperdício de alimentos 


\section{Introdução}

No Brasil, mais de 5,5 milhões de crianças frequentam Centros de Educação Infantil (CEI), os quais englobam creches e pré-escolas que funcionam em período parcial ou integral, perfazendo um total de 4 a 7 horas diáriass ${ }^{1,2}$.

As crianças regularmente matriculadas em CEI são atendidas pelo Programa Nacional de Alimentação Escolar (PNAE), instituído em 1955, que possui como objetivo primário: "contribuir para o crescimento e o desenvolvimento biopsicossocial, a aprendizagem, o rendimento escolar e a formação de hábitos alimentares saudáveis dos alunos, por meio de ações de educação alimentar e nutricional e da oferta de refeições que cubram as suas necessidades nutricionais durante o período letivo"3.

Para o alcance dos objetivos propostos, o PNAE estabelece que os cardápios fornecidos aos CEI, elaborados por nutricionistas, devem contemplar no mínimo 30\% das necessidades nutricionais diárias, distribuídas em, minimamente, duas refeições, quando as crianças permanecem em período parcial e no mínimo 70\%, distribuídas em pelo menos três refeições, para as crianças em período integral ${ }^{4}$.

A concepção do PNAE tem o propósito de respeitar, promover, proteger, facilitar e prover o Direito Humano a Alimentação Adequada (DHAA) para uma parcela transcendente da população. Nesta perspectiva, disseminar e consolidar o sistema de monitoramento e de avaliação somam possibilidades para fazer do Programa um espaço efetivo para a promoção da alimentação saudável e também para a formação de sujeitos de direitos ${ }^{5,6}$.

Diante desta concepção, pesquisadores têmse articulado na tentativa de compreender e clarificar a complexidade e os obstáculos para garantir a efetividade prática das propostas e objetivos previstos pelo PNAE, constatando que o consumo alimentar, quantitativo e qualitativo revela-se como um desafio persistente do Programa a ser enfrentado no âmbito da saúde pública ${ }^{7,8}$.

Diante do exposto, o presente estudo tem como objetivo avaliar a adequação nutricional da alimentação ofertada e consumida por crianças regularmente matriculadas em CEI em Maceió/ $\mathrm{AL}$, bem como quantificar o desperdício de alimentos nestas instituições.

\section{Métodos}

\section{Delineamento do estudo}

Este estudo, transversal, é parte integrante do projeto de pesquisa "Situação nutricional de crianças em creches públicas e ações de alimentação e nutrição na atenção básica: um enfoque intersetorial", realizado em todos os CEI $(\mathrm{n}=5)$ inseridos no sétimo distrito sanitário de saúde de Maceió, representando 10\% do total das instituições municipais $(n=53)$ e $5 \%$ das crianças matriculadas em CEI $(n=6211)$ do município9.

$\mathrm{O}$ estado de Alagoas situa-se na região nordeste do Brasil e integra a agenda prioritária no contexto da Saúde Pública, apresentando incidência de pobreza de $58,37 \%$, e índice de Gini de 0,52 , além de liderar o ranking dos estados mais pobres e com menores índices de desenvolvimento humano, equivalente a $0,72^{10}$. A capital, Maceió, é dividida em oito distritos sanitários, em nossa pesquisa foram estudados apenas os CEI pertencentes ao sétimo distrito sanitário, por se tratar de um dos distritos de maior vulnerabilidade social ${ }^{11}$.

A pesquisa ocorreu entre março e julho de 2014 e foram incluídos os Maternais (I e II) e Jar$\operatorname{dim}(\mathrm{I})$ de todos os CEI $(\mathrm{n}=5)$. Dentre as crianças elegíveis, apenas duas não foram recrutadas devido recusa dos pais/responsáveis em autorizar a participação na pesquisa e outras cinco crianças cujos pais/responsáveis não compareceram para a entrevista. Desta forma, o universo de pesquisa foi composto por 359 crianças de ambos os sexos, com faixa etária entre 17 e 63 meses, que frequentavam regularmente os CEI e cujos pais/ responsáveis assinaram o termo de consentimento livre e esclarecido.

Por questões éticas, os CEI foram identificados mediante enumeração aleatória de 1 a 5 . Todas as instituições funcionavam de segunda a sexta-feira, em tempo integral, todavia com atendimento das crianças em período parcial (manhã ou tarde), com exceção do Maternal I dos CEI 3 e 4 , no qual as mesmas crianças permaneciam nos dois períodos (integral).

\section{Alimentação servida, consumida e desperdício de alimentos}

A equipe, composta por quatro nutricionistas pós-graduandas devidamente treinadas e supervisionadas, acompanhou nos cinco CEI todas as refeições realizadas em cada sala durante três dias não consecutivos, totalizando 15 dias de análise e 
173 refeições, sendo adotado o método de pesagem direta dos alimentos. As coletas ocorreram unicamente em dias letivos, de segunda a sextafeira, não tendo ocorrido nenhuma data festiva ou qualquer outra atividade institucional que implicasse em alteração na rotina alimentar habitual.

Acrescenta-se que os cardápios de todos os CEI eram elaborados por nutricionistas da Secretaria Municipal de Educação (SEMED) e que os CEI estudados possuíam gestão escolarizada da alimentação, processo pelo qual os recursos recebidos do Fundo Nacional de Desenvolvimento da Educação (FNDE) são repassados diretamente às escolas. Nesse tipo de gestão, são as próprias escolas que administram os recursos e se responsabilizam pela compra, recebimento e armazenamento dos gêneros alimentícios, além do preparo, distribuição das refeições e atividades gerenciais.

Durante o preparo das refeições pelas cozinheiras da instituição, foi realizada a pesagem de todos os ingredientes de cada preparação, assim como do rendimento final, como estratégia para favorecer o posterior cálculo nutricional das preparações.

Para a pesagem dos alimentos sólidos foi utilizada uma balança eletrônica digital portátil, calibrada e com capacidade de $5 \mathrm{~kg}$. Os alimentos líquidos foram medidos com o auxílio de recipiente com capacidade para $1.000 \mathrm{~mL}$.

O desperdício quantitativo de alimentos foi determinado pelo Índice de Resto-Ingestão (IR), que consiste na relação entre o alimento que foi rejeitado no prato e a quantidade de alimento distribuído. O peso da refeição distribuída foi determinado após a retirada das partes não comestíveis, como cascas e ossos ${ }^{12}$. Assim estabelece-se a relação percentual entre o Peso da Refeição Rejeitada (PRR) e o Peso da Refeição Distribuída $(P R D)$, representada pela fórmula: $\% I R=P R R x$ 100/PRD ${ }^{13}$ (Quadro 1).

Para a determinação do PRD foram coletadas, no momento da distribuição das refeições, três porções de cada alimento e/ou preparação, selecionados aleatoriamente, para obter a quantidade média servida às crianças. Por conseguinte, multiplicou-se o valor pelo número de crianças de cada sala de aula. As repetições foram somadas ao valor total, obtendo desta forma o PRD ${ }^{13}$.

Foram obtidos os pesos e/ou medidas individuais de cada alimento e/ou preparação, correspondente ao PRR. Acrescenta-se que no almoço e no jantar, sempre que as refeições eram constituídas por diferentes preparações servidas no mesmo prato, a análise considerou o \%IR proporcional para cada preparação ${ }^{12}$. O desperdício alimentar das diferentes preparações foi analisado assumindo-se como aceitável IR inferiores a 10\% ${ }^{4}$.

Para o cálculo nutricional das refeições, foi determinado o per capita servido (PCservido) e consumido (PCconsumido). O PCservido consiste no PRD dividido pelo número de crianças presentes e o PCconsumido foi determinado pelo PRD subtraindo o PRR e dividindo pelo número de crianças presentes. Foi utilizado o software DietWin Profissional $2.0^{\circledast}$.

Posteriormente foi realizado o cálculo da adequação nutricional, tanto do PCservido quanto PConsumido, considerando os valores de referência do PNAE para energia, fibra alimentar, macronutrientes, vitamina A, vitamina $\mathrm{C}$, cálcio, ferro, magnésio e zinco ${ }^{4}$. Ressalta-se que as recomendações nutricionais se diferem para crianças em período parcial e integral, assim como por faixas de idade: um a três anos e quatro a cinco $\operatorname{anos}^{4}$, tendo a análise dos dados considerado estas particularidades.

\section{Análise de dados}

Visando uniformizar o preenchimento de todos os instrumentos, elaborou-se um manual com orientações aos entrevistadores e codificação das variáveis. Os dados coletados foram analisados quanto à sua consistência interna, seguida da dupla digitação, validação e análise estatística no software Epi-Info 7 (Centers for Disease Control and Prevention, Atlanta, EUA).

Quadro 1. Fórmulas dos índices avaliados, Maceió/AL, 2014.

$\%$ IR = PRR x 100/PRD;

$\mathrm{PCservido}=\mathrm{PRD} / \mathrm{n}^{\circ}$ de crianças presentes no momento da refeição;

PCconsumido $=(\mathrm{PRD}-\mathrm{PRR}) / \mathrm{n}^{\circ}$ de crianças presentes no momento da refeição.

$\% \mathrm{IR}=\mathrm{PRR} \times 100 / \mathrm{PRD}$

PCservido $=\mathrm{PRD} / \mathrm{n}^{\circ}$ de crianças presentes no momento da refeição

PCconsumido $=(\mathrm{PRD}-\mathrm{PRR}) / \mathrm{n}^{\circ}$ de crianças presentes no momento da refeição 
Foi realizada análise descritiva, por meio de frequências absolutas e relativas. Posteriormente foi utilizado o teste de Kolmogorov-Smirnov para avaliação da aderência das variáveis quantitativas à distribuição normal. Após análise, as variáveis foram apresentadas na forma de média, desvio-padrão e valores mínimo e máximo. As comparações entre as médias dos nutrientes ofertados e consumidos foram realizadas por meio do teste t de Student, adotando-se, como nível de significância estatística, o valor de $\mathrm{p}<0,05$.

Acrescenta-se que todos os participantes consentiram com a participação na pesquisa mediante assinatura do Termo de Consentimento Livre e Esclarecido, tendo o projeto sido aprovado pelo Comitê de Ética em Pesquisa da UFAL.

\section{Resultados}

A idade média das 359 crianças estudadas foi de 45 meses, com distribuição similar da frequência entre os sexos. O número de crianças e as respectivas idades médias de cada instituição foram: CEI 1 ( $\mathrm{n}=32 ; 46,5$ meses), CEI 2 ( $\mathrm{n}=71 ; 48$ meses), CEI 3 ( $\mathrm{n}=123 ; 36,5$ meses), CEI 4 ( $\mathrm{n}=$ 42; 41 meses), CEI 5 ( $\mathrm{n}=91 ; 51$ meses).

Das 173 refeições analisadas, constatou-se variação nos horários de início e término, entre as salas e instituições. O lanche da manhã foi servido entre $07 \mathrm{~h} 30 \mathrm{~min}$ e $9 \mathrm{~h} 30 \mathrm{~min}$, almoço entre $10 \mathrm{~h} 40 \mathrm{~min}$ e $11 \mathrm{~h}$, lanche da tarde entre $13 \mathrm{~h} \mathrm{e}$ $13 \mathrm{~h} 45 \mathrm{~min}$ e o jantar entre $15 \mathrm{~h}$ e $16 \mathrm{~h} 30 \mathrm{~min}$. Ademais as cinco instituições adotavam o mesmo cardápio para todas as crianças, independentemente da idade, nível escolar ou tempo de permanência (parcial ou integral).

A Tabela 1 apresenta os alimentos/preparações que foram ofertados, na qual verifica-se que o CEI 1 ofertou apenas uma refeição por período: lanches da manhã e tarde. Nos demais foram ofertadas duas refeições: lanche da manhã e almoço, no período matutino e lanche da tarde e jantar no período vespertino.

No que concerne à constituição das refeições, nos lanches da manhã e da tarde constata-se a oferta de leite (com achocolatado) ou preparações a base de leite (mingau de amido de milho, munguzá, cuscuz com leite, vitamina de frutas) e pão (com proteína texturizada de soja), alternando-se biscoitos doces e salgados. No almoço e jantar foram oferecidos arroz e feijão ou macarrão ou inhame com carne bovina, de frango, ovo ou proteína texturizada de soja. Cabe destacar a substituição do lanche por risoto de frango e ar- roz branco com carne (CEI 1) e almoço e jantar por biscoito acompanhado de suco de fruta, arroz doce (CEI 5) e munguzá (CEI 4).

Dos 85 alimentos/preparações presentes no cardápio, $68 \%$ apresentou percentual de resto -ingestão (\%IR), superior a 10\% (Tabela 1). A média total de desperdício por refeição revelou que de $79 \%$ a $84 \%$ dos alimentos servidos foram efetivamente consumidos pelas crianças.

A Tabela 2 contempla as médias dos nutrientes servidos e consumidos, com os respectivos desvios padrão, valores mínimos e máximos, adequação nutricional e valores de $\mathrm{p}$ das diferenças. Constatase, que nos CEI que atendem em período integral, tanto as refeições servidas como consumidas atenderam menos de $40 \%$ dos valores preconizados, com exceção da vitamina C. De forma semelhante, nas instituições que atendiam parcialmente, a oferta e consumo de todos os nutrientes foram inferiores ao recomendado, exceto para magnésio, zinco e vitamina C, cuja oferta e consumo foi cerca de 5 a 6 vezes superior ao estipulado.

Analisando comparativamente as médias dos nutrientes servidos e consumidos, verifica-se diferença estatisticamente significante $(\mathrm{p}<0,05)$ no consumo de fibra alimentar, vitamina $\mathrm{A}$, vitamina $\mathrm{C}$, cálcio, ferro e zinco entre as crianças de 1 a 3 anos, atendidas em período integral, e no período parcial para vitamina A e zinco.

\section{Discussão}

Este estudo avaliou o consumo alimentar das crianças matriculadas em CEI, a fim de analisar a quantidade de nutrientes ofertados às mesmas e a quantidade efetivamente consumida, além de quantificar o desperdício alimentar por meio do $\%$ IR.

Analisando-se os dados apresentados, constata-se que a composição do cardápio apresentou pouca variedade, com acentuada monotonia de alimentos e preparações, destacando-se a ausência de verduras e legumes em todos os dias e instituições avaliadas. Adicionalmente, as frutas in natura compuseram $16,6 \%(\mathrm{n}=9)$ refeições, com pouca variação, restringindo-se a oferta de melancia, banana, maçã e laranja lima.

Tais alimentos integram a base para o alcance de uma alimentação nutricionalmente balanceada, saborosa, culturalmente apropriada e promotora de um sistema alimentar sustentável. A variedade dentro de um mesmo grupo de alimentos, além de implicar em maior diversidade de nutrientes, proporciona variações sensoriais, 
Tabela 1. Alimentos ofertados para crianças matriculadas em CEIs† e o percentual de Resto- Ingestão (RI) ‡ das refeições em Maceió (AL), Brasil, 2014.

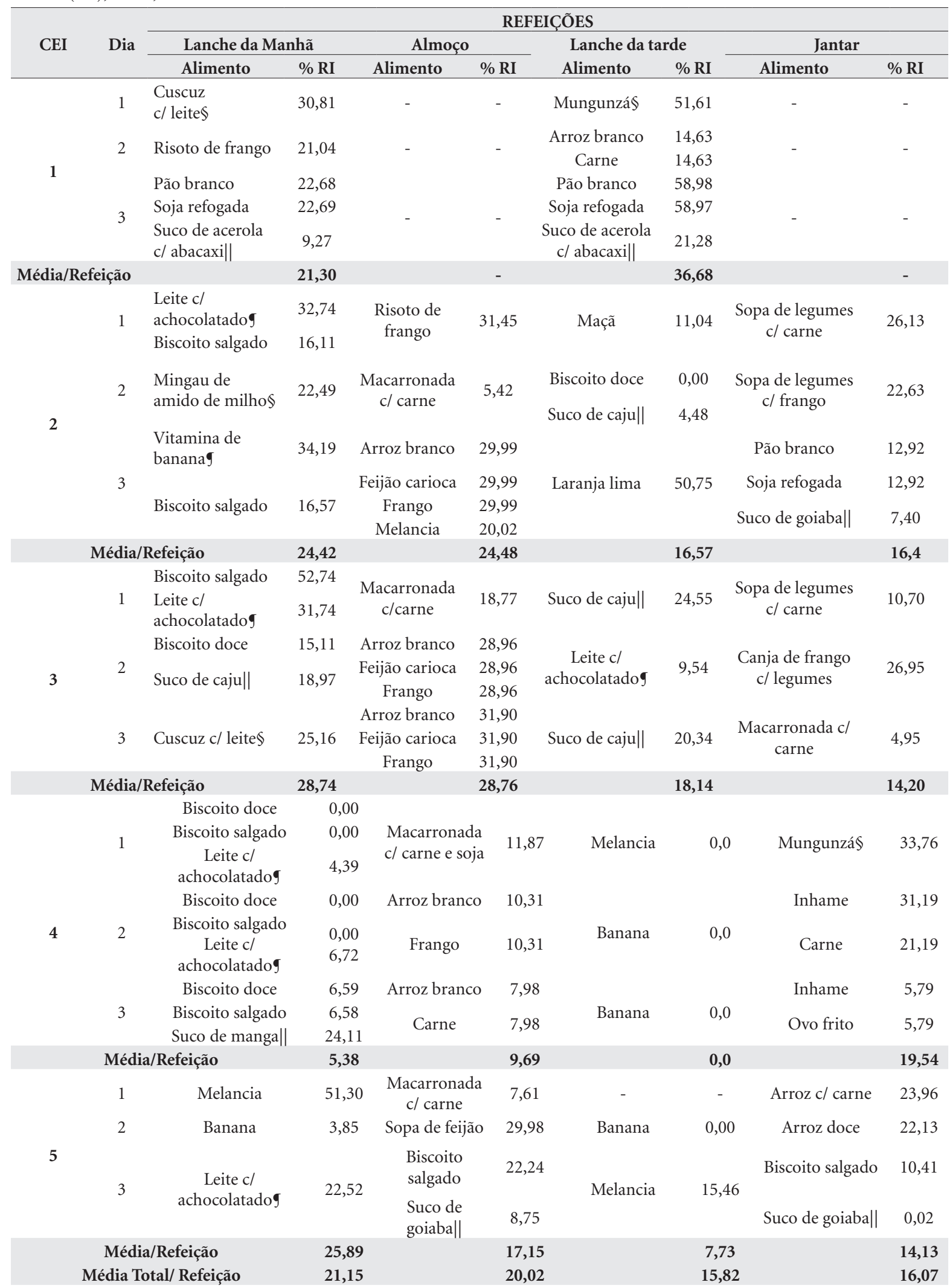

¥\%IR = Peso da Refeição Rejeitada (PRR) x 100/Peso da Refeição Distribuída (PRD); † Centro de Educação Infantil; § Preparações elaboradas à base de milho; || Preparação elaborada com polpa de fruta e adição de açúcar; 9 Preparações com adição de açúcar. 
Tabela 2. Adequação de energia e nutrientes, média, desvio padrão, valor mínimo e valor máximo servido e consumido por crianças frequentadoras de CEIs† em Maceió (AL), Brasil, 2014.

\begin{tabular}{|c|c|c|c|c|c|c|}
\hline & \multirow[b]{2}{*}{$\begin{array}{c}\text { Recomendação } \\
\text { Diária }^{3}\end{array}$} & \multicolumn{2}{|c|}{ Servido } & \multicolumn{3}{|c|}{ Consumido } \\
\hline & & $\begin{array}{c}\text { Média ( } \pm \mathrm{DP}) \\
\text { (Mín./Máx.) }\end{array}$ & $\begin{array}{c}\text { Adequação } \\
(\%)\end{array}$ & $\begin{array}{c}\text { Média }( \pm \mathrm{DP}) \\
(\text { Mín/Max })\end{array}$ & $\begin{array}{c}\text { Adequação } \\
(\%)\end{array}$ & $\mathbf{p}^{*}$ \\
\hline \multicolumn{7}{|c|}{ Período integral - crianças de 1 a 3 anos } \\
\hline Energia (Kcal) & 700,00 & $\begin{array}{c}128,16( \pm 51,24) \\
(27,14 / 254,10)\end{array}$ & 18,31 & $\begin{array}{c}104,26( \pm 43,71) \\
(27,14 / 235,46)\end{array}$ & 14,89 & 0,188 \\
\hline Carboidrato $(\mathrm{g})$ & 114,90 & $\begin{array}{c}19,62( \pm 39,14) \\
(6,11 / 39,14)\end{array}$ & 17,08 & $\begin{array}{c}16,18( \pm 6,55) \\
(6,11 / 36,25)\end{array}$ & 14,08 & 0,166 \\
\hline Proteína $(g)$ & 21,90 & $\begin{array}{l}6,10( \pm 5,19) \\
(0,00 / 20,08)\end{array}$ & 27,85 & $\begin{array}{l}4,81( \pm 4,04) \\
(0,00 / 18,57)\end{array}$ & 21,96 & 0,084 \\
\hline Lipídio (g) & 17,50 & $\begin{array}{c}2,81( \pm 2,10) \\
(0,00 / 7,20)\end{array}$ & 16,06 & $\begin{array}{c}2,24( \pm 1,73) \\
(0,00 / 6,63)\end{array}$ & 12,80 & 0,052 \\
\hline Fibra $(g)$ & 13,30 & $\begin{array}{c}1,29( \pm 1,09) \\
(0,08 / 5,02)\end{array}$ & 9,70 & $\begin{array}{c}1,03( \pm 0,82) \\
(0,08 / 4,67)\end{array}$ & 7,74 & $<0,05$ \\
\hline Vitamina A ( $\mu \mathrm{g})$ & 210,00 & $\begin{array}{c}35,42( \pm 43,94) \\
(0,00 / 138,00)\end{array}$ & 16,87 & $\begin{array}{c}28,77( \pm 37,99) \\
(0,00 / 129,00)\end{array}$ & 13,70 & $<0,05$ \\
\hline Vitamina C (mg) & 12,00 & $\begin{array}{c}13,93( \pm 23,77) \\
(0,00 / 82,63)\end{array}$ & 116,08 & $\begin{array}{c}11,94( \pm 20,90) \\
(00,00 / 73,50)\end{array}$ & 99,50 & $<0,05$ \\
\hline Cálcio (mg) & 350,00 & $\begin{array}{c}40,85( \pm 59,81) \\
(3,99 / 202,34)\end{array}$ & 11,67 & $\begin{array}{c}31,60( \pm 48,32) \\
(3,63 / 202,34)\end{array}$ & 9,03 & $<0,05$ \\
\hline Ferro (mg) & 4,90 & $\begin{array}{c}0,68 \\
(0,07 / 2,18)\end{array}$ & 13,88 & $\begin{array}{c}0,55( \pm 0,43) \\
(0,05 / 2,01)\end{array}$ & 11,22 & $<0,05$ \\
\hline Magnésio (mg) & 56,00 & $\begin{array}{c}22,07( \pm 0,53) \\
(0,13 / 86,06)\end{array}$ & 39,41 & $\begin{array}{c}17,26( \pm 15,23) \\
(0,09 / 79,58)\end{array}$ & 30,82 & 0,060 \\
\hline Zinco(mg) & 2,10 & $\begin{array}{c}0,71( \pm 0,80) \\
(0,00 / 3,32)\end{array}$ & 33,81 & $\begin{array}{l}0,59( \pm 0,73) \\
(00,00 / 3,07)\end{array}$ & 28,10 & $<0,05$ \\
\hline \multicolumn{7}{|c|}{ Período parcial - crianças de 1 a 3 anos } \\
\hline Energia (Kcal) & 300,0 & $\begin{array}{c}181,69( \pm 70,48) \\
(50,16 / 342,16)\end{array}$ & 60,56 & $\begin{array}{c}144,16( \pm 65,32) \\
(19,49 / 299,54)\end{array}$ & 48,05 & 0,377 \\
\hline Carboidrato $(\mathrm{g})$ & 48,8 & $\begin{array}{c}29,22( \pm 10,78) \\
(11,29 / 53,95)\end{array}$ & 59,88 & $\begin{array}{c}23,42( \pm 10,78) \\
(4,38 / 49,57)\end{array}$ & 47,99 & 0,368 \\
\hline Proteína $(g)$ & 9,4 & $\begin{array}{l}7,70( \pm 5,52) \\
(0,83 / 20,08)\end{array}$ & 81,91 & $\begin{array}{l}5,97( \pm 4,51) \\
(0,49 / 17,69)\end{array}$ & 63,51 & 0,282 \\
\hline Lipídio (g) & 7,5 & $\begin{array}{c}3,77( \pm 2,63) \\
(0,00 / 9,45)\end{array}$ & 50,27 & $\begin{array}{c}2,95( \pm 2,09) \\
(0,00 / 7,73)\end{array}$ & 39,33 & 0,132 \\
\hline Fibra (g) & 5,7 & $\begin{array}{c}1,76( \pm 1,38) \\
(0,00 / 7,28)\end{array}$ & 30,88 & $\begin{array}{c}1,37( \pm 1,02) \\
(0,00 / 4,63)\end{array}$ & 24,04 & 0,127 \\
\hline Vitamina A $(\mu \mathrm{g})$ & 90,0 & $\begin{array}{c}52,60( \pm 59,69) \\
(0,00 / 300,46)\end{array}$ & 58,44 & $\begin{array}{c}41,40( \pm 48,22) \\
(0,00 / 231,90)\end{array}$ & 46,00 & $<0,05$ \\
\hline Vitamina C (mg) & 5,0 & $\begin{array}{c}32,65( \pm 63,02) \\
(0,00 / 261,87)\end{array}$ & 653,00 & $\begin{array}{c}28,27( \pm 56,51) \\
(0,00 / 234,25)\end{array}$ & 565,40 & $<0,05$ \\
\hline Cálcio (mg) & 150,0 & $\begin{array}{c}66,22( \pm 82,87) \\
(5,13 / 309,55)\end{array}$ & 44,15 & $\begin{array}{c}49,38( \pm 61,72) \\
(4,25 / 238,94)\end{array}$ & 32,92 & 0,297 \\
\hline Ferro $(\mathrm{mg})$ & 2,1 & $\begin{array}{c}1,08( \pm 0,89) \\
(0,22 / 5,30)\end{array}$ & 51,43 & $\begin{array}{c}0,85( \pm 0,71) \\
(0,11 / 4,09)\end{array}$ & 40,48 & 0,090 \\
\hline Magnésio (mg) & 24,0 & $\begin{array}{c}36,06( \pm 26,51) \\
(0,13 / 114,64)\end{array}$ & 150,25 & $\begin{array}{c}27,53( \pm 21,03) \\
(0,07 / 99,70)\end{array}$ & 114,71 & 0,294 \\
\hline Zinco(mg) & 0,9 & $\begin{array}{c}0,99( \pm 1,03) \\
(0,00 / 4,50)\end{array}$ & 110,00 & $\begin{array}{c}0,80( \pm 0,93) \\
(0,00 / 4,02)\end{array}$ & 88,89 & $<0,05$ \\
\hline
\end{tabular}

por propiciar diversificação de sabores, aromas, cores e texturas ${ }^{14}$.

Neste contexto, em ensaio clínico envolvendo pré-escolares, Roe et al. ${ }^{15}$ investigaram se a dispo- nibilidade de opções variadas de frutas e vegetais na mesma refeição aumentava a seleção e ingestão destes alimentos, quando comparadas a oferta de apenas uma. Os pesquisadores concluíram que 
Tabela 2. Adequação de energia e nutrientes, média, desvio padrão, valor mínimo e valor máximo servido e consumido por crianças frequentadoras de CEIs† em Maceió (AL), Brasil, 2014.

\begin{tabular}{|c|c|c|c|c|c|c|}
\hline & \multirow[b]{2}{*}{$\begin{array}{c}\text { Recomendação } \\
\text { Diária }^{3}\end{array}$} & \multicolumn{2}{|c|}{ Servido } & \multicolumn{3}{|c|}{ Consumido } \\
\hline & & $\begin{array}{c}\text { Média }( \pm \mathrm{DP}) \\
\text { (Mín./Máx.) }\end{array}$ & $\begin{array}{l}\text { Adequação } \\
(\%)\end{array}$ & $\begin{array}{c}\text { Média }( \pm \mathrm{DP}) \\
(\text { Mín/Max) }\end{array}$ & $\begin{array}{l}\text { Adequação } \\
(\%)\end{array}$ & $\mathbf{p}^{*}$ \\
\hline \multicolumn{7}{|c|}{ Período parcial - crianças de 4 a 5 anos } \\
\hline Energia (Kcal) & 400,0 & $\begin{array}{c}128,01( \pm 73,46) \\
(34,92 / 294,14)\end{array}$ & 32,00 & $\begin{array}{c}102,95( \pm 67,17) \\
(19,25 / 294,14)\end{array}$ & 25,74 & 0,427 \\
\hline Carboidrato (g) & 65,0 & $\begin{array}{c}21,87( \pm 13,78) \\
(5,38 / 56,24)\end{array}$ & 33,65 & $\begin{array}{c}17,53( \pm 13,00) \\
(2,78 / 48,79)\end{array}$ & 26,97 & 0,424 \\
\hline Proteína $(g)$ & 12,5 & $\begin{array}{l}5,06( \pm 4,31) \\
(0,00 / 16,46)\end{array}$ & 40,48 & $\begin{array}{l}4,04( \pm 3,79) \\
(0,00 / 16,46)\end{array}$ & 32,32 & 0,181 \\
\hline Lipídio (g) & 10,0 & $\begin{array}{c}2,22( \pm 2,09) \\
(0,00 / 8,64)\end{array}$ & 22,20 & $\begin{array}{c}1,76( \pm 1,61) \\
(0,00 / 5,54)\end{array}$ & 17,60 & 0,122 \\
\hline Fibra $(g)$ & 7,5 & $\begin{array}{c}1,51( \pm 0,98) \\
(0,10 / 4,53)\end{array}$ & 20,13 & $\begin{array}{c}1,78( \pm 0,86) \\
(0,08 / 3,98)\end{array}$ & 23,73 & 0,299 \\
\hline Vitamina A $(\mu \mathrm{g})$ & 120,0 & $\begin{array}{c}19,77( \pm 32,28) \\
(0,00 / 127,50)\end{array}$ & 16,48 & $\begin{array}{c}15,98( \pm 26,84) \\
(0,00 / 118,89)\end{array}$ & 13,32 & $<0,05$ \\
\hline Vitamina C (mg) & 8,0 & $\begin{array}{c}25,80( \pm 32,63) \\
(0,00 / 126,07)\end{array}$ & 322,50 & $\begin{array}{c}19,02( \pm 27,55) \\
(0,00 / 126,07)\end{array}$ & 237,75 & 0,332 \\
\hline Cálcio (mg) & 240,0 & $\begin{array}{c}33,44( \pm 47,08) \\
(1,55 / 202,34)\end{array}$ & 13,93 & $\begin{array}{c}25,84( \pm 39,13) \\
(1,23 / 188,71)\end{array}$ & 10,77 & 0,422 \\
\hline Ferro $(\mathrm{mg})$ & 3,0 & $\begin{array}{c}0,69( \pm 0,52) \\
(0,05 / 2,30)\end{array}$ & 23,00 & $\begin{array}{c}0,57( \pm 0,50) \\
(0,04 / 2,30)\end{array}$ & 19,00 & 0,265 \\
\hline Magnésio (mg) & 39,0 & $\begin{array}{c}18,00( \pm 15,19) \\
(0,16 / 67,06)\end{array}$ & 46,15 & $\begin{array}{c}14,08( \pm 12,70) \\
(0,08 / 57,26)\end{array}$ & 36,10 & 0,344 \\
\hline Zinco(mg) & 1,5 & $\begin{array}{c}0,62( \pm 0,93) \\
(0,00 / 3,85)\end{array}$ & 41,33 & $\begin{array}{l}0,53( \pm 0,8) \\
(0,00 / 3,85)\end{array}$ & 35,33 & $<0,05$ \\
\hline
\end{tabular}

Recomendação diária de acordo com os valores estabelecidos pelo PNAE ${ }^{3}$ † Centro de Educação Infantil; $\ddagger$ Desvio-Padrão; ${ }^{\star}$ Teste $t$ de stundent.

quando a oferta foi variada, houve seleção e consumo de ao menos um dos alimentos por $94 \%$ das crianças, representando um aumento, estatisticamente significante, na probabilidade de seleção.

Além da diversidade dos itens alimentares, acrescenta-se como estratégia para contornar a natureza repetitiva e exposição a alimentos muito similares, a adoção de técnicas de preparo diferenciadas, favorecendo a redução do desperdício alimentar ${ }^{15,16}$.

A baixa aceitação dos alimentos identificada em nosso estudo é comprovada pelos valores percentuais de IR identificados, os quais, para $68 \%$ dos alimentos ofertados, variaram de 10 a 59\%. Tais resultados parecem refletir uma realidade, de certa forma homogênea, no contexto das creches públicas inseridas em diferentes cenários geográficos. A exemplificar, em outro estudo que realizamos com 366 crianças de 12 a 36 meses frequentadoras de creches públicas de São Paulo/SP, adotando metodologia equivalente, encontramos variação entre 11 e $47 \%$ do percentual de Resto-Ingestão ${ }^{16}$.
Blondin et al. ${ }^{17}$ avaliaram qualitativamente o desperdício de alimentos entre pré-escolares atendidos por um programa universal gratuito, o School Breakfast Program, e identificaram as seguintes justificativas para as crianças não selecionarem os alimentos ou deixarem-nos no prato: quantidade excessiva de alimento servido, ausência de fome, não apreciar as opções de alimentos ou a realização de refeição prévia no domicílio.

O elevado desperdício de alimentos pode ser influenciado pela falta de padronização das preparações, inexistência de fichas técnicas e omissão da aplicação de testes de aceitabilidade ${ }^{18}$.

De modo suplementar, retrata a necessidade emergencial de capacitações voltadas as todos os profissionais, cozinheiros, diretores e educadores, abrangendo desde o planejamento para garantia da execução do cardápio, aprimoramento de técnicas culinárias enfocadas na variação das formas de preparo, texturas, apresentações visuais, sabor e estratégias adequadas de incentivo durante a oferta, como forma de despertar o interesse das 
crianças e promover maior aceitação dos alimentos e preparações ${ }^{18}$.

Aprofundando-se na análise do Resto-Ingestão identificada em nosso estudo, desperta interesse o fato de terem apresentado baixa aceitação duas preparações que compõem a cultura alimentar típica desta região brasileira, cuscuz com leite, cujo IR variou de 25,2 a 30,8\%, e munguzá, cujo IR variou de 33,8 a 51,6\%. Estes resultados podem ser justificados pelas novas tendências de consumo alimentar no Brasil, que integram o ideograma da transição alimentar e nutricional, e que se caracterizam pela substituição de alimentos tradicionais por ultraprocessados entre as famílias brasileiras, disseminada nas diferentes classes socioecômicas e regionais ${ }^{14,19}$.

Tradicionalmente na região Nordeste, o "cuscuz com leite" consiste em uma preparação elaborada com farinha de milho e leite e açúcar refinado e o "munguzá", que em outras regiões brasileiras é denominado como "canjica”, é preparado com o grão de milho, leite, leite de coco, açúcar refinado e especiarias (canela e cravo). O milho e açúcar são alimentos presentes na culinária brasileira, correspondendo ao País que mais consome açúcar no mundo, com ingestão per capita anual estimada em $55 \mathrm{~kg}$, sendo a média mundial por habitante equivalente a $21 \mathrm{~kg}^{20}$.

Adicionalmente, além destes alimentos serem economicamente de fácil acesso e predominantes nas preparações típicas da região Nordeste, a monocultura de cana-de-açúcar presente por muitos anos nesta região também parece refletir no seu maior consumo ${ }^{21,22}$.

Em concordância com os nossos achados, Chaves et al. ${ }^{22}$ investigaram o cardápio da alimentação escolar fornecida nas distintas regiões do Brasil (Norte, Nordeste, Sul, Sudeste e Centro -oeste), e identificaram que na região nordeste, as preparações mais frequentes foram: cuscuz (29\%), munguzá (16\%), arroz doce (8\%) e mingau de milho $(8 \%)$.

No entanto, deve-se ressaltar que o PNAE recomenda que o açúcar adicionado às preparações deve se limitar a $10 \%$ da energia total ${ }^{4}$, e que a oferta de preparações acrescidas de açúcar deve ser evitada, devendo-se acrescentar frutas e hortaliças ao preparo, no intuito de enriquecer a dieta com micronutrientes ${ }^{23}$. O Programa recomenda ainda que a oferta de doces, categoria na qual se insere o munguzá, deve ser limitada a duas porções por semana, equivalendo ao máximo de 110 kcal/porção.

Adicionalmente cumpre enfatizar que a composição nutricional das preparações munguzá e cuscuz com leite, cuja oferta foi observada também no jantar, não condiz com as expectativas da composição desta refeição, que se presume ser completa, em termos de composição de grupos alimentares e nutrientes ${ }^{4,14}$.

No que concerne ao número de refeições diárias, observou-se que no CEI 1, que atendia em período parcial, ocorreu a oferta de apenas uma refeição, o que contraria a estipulação do PNAE, que preconiza o mínimo de duas refeições em creches. Tal recomendação assume que garantir a oferta adequada de nutrientes por meio de uma única refeição consiste em tarefa de difícil exequibilidade, considerando a capacidade gástrica das crianças e as possibilidades relacionadas à densidade energética dos alimentos ofertados ${ }^{14,24}$.

Destarte, este cenário retrata a violação do DHAA e a necessidade de os usuários do Programa estarem cientes de que a alimentação escolar é um direito constitucional, além de refletir que as políticas e programas de segurança alimentar e nutricional parecem ainda ser executados e compreendidos sob a perspectiva de "favores". Reforça-se assim a importância dos Conselhos de Alimentação Escolar (CAE) no acompanhamento e fiscalização do programa ${ }^{3,6}$.

Sob este enfoque, em nosso estudo, a magnitude do desperdício alimentar é reafirmada ao se observar a baixa adequação de praticamente a totalidade dos nutrientes avaliados. A energia ofertada correspondeu a $18,3 \%$ do total previsto pelo PNAE para o período integral.

Embora seja previsto o compartilhamento da responsabilidade alimentar com os pais, havendo previsão da realização de pelo menos uma refeição no domicílio, pode-se pressupor a impossibilidade da garantia do suprimento energético adicional das $600 \mathrm{kcal}$ que deixaram de ser consumidas no CEI. Do mesmo modo, os períodos parciais, de 1 a 3 anos e 4 a 5 anos, ofertaram $60,6 \%$ e $32,0 \%$, respectivamente, da energia total preconizada.

Diante do baixo aporte energético, os macronutrientes, estiveram aquém da recomendação, sendo entre as crianças que permaneciam em período integral, a menor oferta de lipídios, com adequação de $16,1 \%$. Para os períodos parciais a oferta de proteínas foi a que mais se aproximou dos valores recomendados.

A adequação percentual do consumo de fibra alimentar variou de 9,7 a 30,9\%, semelhante a outros estudos ${ }^{25-28}$, o que representa fator de risco para constipação intestinal, que afeta muitas crianças em países ocidentais e pode ter efeito significativo no bem-estar infantil ${ }^{27}$. 
Este achado pode estar associado à baixa oferta de frutas e ausência de verduras, legumes e grãos integrais, importantes fontes de fibra alimentar, contrariando a recomendação do PNAE, que prevê a oferta de, no mínimo, três porções de frutas e hortaliças por semana (200g/criança/ semana $)^{4}$.

No que concerne aos micronutrientes, à adequação do cálcio servido variou entre 11,7 a 44,1\%. Similarmente, Lyn et al. ${ }^{26}$, avaliaram 120 cardápios em 24 creches da Geórgia, Estados Unidos, constatando que os mesmos proporcionavam apenas metade das recomendações diárias de cálcio.

Acrescenta-se que em nosso estudo, para o período integral, a diferença entre a quantidade de cálcio servido e consumido foi estatisticamente significante $(\mathrm{p}<0,05)$, podendo ser justificada pelo elevado desperdício de leite e preparações a base de leite, cujo índice de resto-ingestão alcançou valores superiores a $50 \%$.

A baixa aceitação de leite no contexto escolar é encontrada em outras pesquisas ${ }^{16,29,30}$. Henry et al. ${ }^{30}$ investigaram o consumo de leite por crianças canadenses, comparando a aceitação do leite puro com a do leite com achocolatado, concluindo que a sua ingestão reduziu 12,3\% quando o achocolatado foi removido do leite $(26,6 \% \pm$ $5,2 \%$ para $14,31 \% \pm 1,6 \%, \mathrm{p}<0,001)$. Em nosso estudo o leite puro não compôs o cardápio dos CEI, porém o leite com achocolatado alcançou percentual de desperdício de 32,7\%.

Com relação ao ferro, tanto a oferta quanto a ingestão foram acentuadamente inferiores ao preconizado pelo Programa, o que corrobora com outros estudos ${ }^{26,31}$. Tal constatação contribui para o incremento da carência nutricional de maior magnitude mundial, a anemia ferropriva, que acomete $18,1 \%$ das crianças menores de 5 anos no mundo ${ }^{32}$.

A inadequação de ferro encontrada pode ser justificada pela baixa frequência da oferta de carnes, alimento considerado a melhor fonte deste nutriente e que não compôs o cardápio diário das instituições. Em adição, quando presente, a carne integrou preparações em que não é o ingrediente principal, como risotos e macarronadas, em decorrência provável de seu custo elevado, quando comparados aos outros itens alimentares ${ }^{33}$.

Em relação a vitamina $A$, o percentual de adequação não alcançou $45 \%$ da recomendação proposta, fato preocupante quando considerado que a sua deficiência também está entre as carências nutricionais mais prevalentes ${ }^{32}$. Em estudo realizado na região semiárida do estado de Ala- goas com crianças menores de cinco anos, a prevalência de hipovitaminose A encontrada foi de 45,4\% $\%^{34}$. Dados da Pesquisa Nacional de Demografia e Saúde da Criança e da Mulher revelaram que 19\% das crianças menores de cinco anos da região nordeste apresentam níveis séricos reduzidos de vitamina $\mathrm{A}^{35}$.

No que concerne ao zinco, a priori constatou-se entre as crianças de 1 a 3 anos (período parcial) que a oferta foi superior a recomendação (1,0 mg), com adequação de $110 \%$, entretanto o consumo final correspondeu a $88,9 \%(0,8 \mathrm{mg})$ do recomendado. Para os demais períodos o consumo não atingiu metade do esperado, sendo a diferença entre o zinco ofertado e consumido estatisticamente significante $(\mathrm{p}<0,05)$.

Estimativas indicam que $17 \%$ da população mundial estão em risco de deficiência de zinco, considerada um problema de saúde pública frequentemente subnotificado, e que se associa ao retardo do crescimento, comprometimento imunológico, cognitivo e distúrbio neuro-sensorial ${ }^{32,36-38}$.

Diante dos resultados apresentados e discutidos, nosso estudo expõe algumas reflexões acerca da alimentação no contexto das creches e pré-escolas, ao evidenciar que os alimentos ofertados às crianças não atingem as recomendações nutricionais preconizadas pelo PNAE, o que diante da baixa aceitação dos alimentos, evidenciada pelo elevado percentual de Resto-Ingestão, agrava a adequação dos nutrientes efetivamente consumidos pelas crianças.

O Brasil possui um dos mais antigos Programas de Alimentação Escolar do mundo, cujas normas e recomendações são frequentemente avaliadas por profissionais experts em nutrição. No entanto, após sessenta anos da sua existência, prevalece o enorme desafio em alcançar a sua recomendação na ponta final, ou seja, garantir que a criança seja contemplada com os nutrientes em quantidades adequadas, ofertados por meio de alimentos que atendam aos princípios de uma alimentação adequada e saudável ${ }^{39}$.

Embora legalmente as ações de alimentação escolar devam ser supervisionadas por nutricionistas cadastrados no PNAE, prevendo-se ao menos um profissional para cada 500 crianças, $20 \%$ dos municípios brasileiros não possuem nutricionistas atuantes no Programa ${ }^{40}$.

Especificamente em Maceió, onde nosso estudo foi realizado, há sete nutricionistas para atender aos mais de 56 mil estudantes de todos os níveis de educação, dos quais sete mil no contexto dos CEI. Na prática tem-se, portanto, um nutricionista para mais de oito mil crianças, razão 
16 vezes superior ao estipulado pela legislação ${ }^{41}$. Tal situação certamente contribui para a inadequação aqui relatada.

No tocante das limitações do estudo, embora não tenha representatividade nacional, reflete a realidade das crianças que frequentam instituições públicas no estado de Alagoas, considerando o seu perfil socioeconômico e demográfico homogêneo. Outra limitação é que os comportamentos e atitudes dos cuidadores no momento das refeições, como incentivadores e estimuladores do consumo alimentar, não foram investigados.

\section{Conclusões}

Os resultados deste estudo mostraram que tanto os alimentos servidos quanto os efetivamente consumidos não garantiram o atendimento das recomendações nutricionais preconizadas, cons- tatando-se elevada quantidade de alimentos desperdiçados, com diferença estatisticamente significante entre nutrientes específicos ofertados e consumidos.

Assim no intuito de direcionar sugestões de pesquisas de intervenção futuras, que objetivem reverter a situação problemática aqui apresentada, sugere-se como estratégia a reestruturação dos cardápios dos CEI, aumentando a oferta de frutas, legumes, verduras e grãos integrais, inserindo preparações diversificadas e tornando as refeições mais atrativas.Reforça-se ainda a importância da articulação entre nutricionistas, cozinheiros e demais profissionais envolvidos na aquisição, preparo, porcionamento e distribuição das refeições, a fim de aprimorar o planejamento, execução e monitoramento do cardápio, primando pela participação da comunidade no controle social e no acompanhamento das ações para garantir a oferta da alimentação escolar saudável e adequada.

\section{Colaboradores}

CAN Souza, G Longo-Silva, RCE Menezes, AC Araujo, MHA Toloni e MAC Oliveira participaram da concepção do projeto, análise dos dados e redação final do artigo. 


\section{Referências}

1. Brasil. Ministério da Educação (MEC). MEC divulga resultados preliminares do Censo Escolar. [acessado 2016 Out 10]. Disponível em: http://www.brasil.gov.br/educacao/2015/10/mec-divulga-resultados-preliminaresdo-censo-escolar.

2. Brasil. Ministério da Educação (MEC). Diretrizes curriculares nacionais para a educação infantil. Brasília: MEC; 2010

3. Brasil. Lei $n^{\circ} 11.947$, de 16 de junho de 2009. Dispõe sobre o atendimento da alimentação escolar e do Programa Dinheiro Direto na Escola aos alunos da educação básica; altera as Leis nos 10.880 , de 9 de junho de 2004, 11.273, de 6 de fevereiro de 2006, 11.507, de 20 de julho de 2007; revoga dispositivos da Medida Provisória no 2.178-36, de 24 de agosto de 2001, e a Lei no 8.913, de 12 de julho de 1994; e dá outras providências. Diário Oficial da União 2009; 17 jun.

4. Brasil. Resolução no 26, de 17 de junho de 2013. Dispõe sobre o atendimento da alimentação escolar aos alunos da educação básica no âmbito do Programa Nacional de Alimentação Escolar - PNAE. Diário Oficial da União 2013; 18 jun.

5. Brasil. Lei $n^{\circ} 11.346$, de 15 de setembro de 2006. Cria o Sistema Nacional de Segurança Alimentar e Nutricional - SISAN com vistas em assegurar o direito humano à alimentação adequada e dá outras providências. 2006. Diário Oficial da União 2006; 18 set.

6. Valente FLS, Franceschini T, Burity V. A Exigibilidade do Direito Humano à Alimentação Adequada. Brasília: ABRANDH, FAO; 2007.

7. Neelon SEB, Copeland KA, Ball SC, Bradley L, Ward DS. Comparison of Menus to Actual Foods and Beverages Served in North Carolina Child-Care Centers. J Am Diet Assoc 2010; 110(12):1890-1895.

8. Molloy CJ, Kearney J, Hayes N, Slattery CG, Corish C. Healthy incentive scheme in the Irish full-day-care preschool setting. Proc Nutr Soc 2014; 73(1):147-158.

9. Brasil. Fundo Nacional de Desenvolvimento da Educação (FNDE). Alunado por ação do programa nacional de alimentação escolar. [acessado 2016 Nov 01]. Disponível em: http://www.fnde.gov.br/pnaeweb/publico/ relatorioDelegacaoEstadual.do

10. Instituto Brasileiro de Geografia e Estatística (IBGE). Censo Demográfico 2010. [acessado 2016 Out 28]. Disponível em: http://www.ibge.gov.br/cidadesat/link.php? $\mathrm{uf}=\mathrm{al}$

11. Brasil. Ministério do Desenvolvimento Social e Agrário. Mapas Temáticos de Vulnerabilidade Social. [acessado 2016 Out 31]. Disponível em: http://aplicacoes.mds. gov.br/sagirmps/mapas_pobreza_mapa/cifam.php

12. Vaz CS. Alimentação de coletividade: uma abordagem gerencial. Manual Prático do Gestor de Serviços de Refeições Coletivas. Brasília: Metha; 2003.

13. Carver AF, Patton MB. Plate waste in a school lunch. I. Over-all waste. J Am Diet Assoc 1958; 34(6):615-618.

14. Brasil. Ministério da Saúde (MS). Guia alimentar para a população brasileira. Brasília: MS; 2014.

15. Roe LS, Meengs JS, Birch LL, Rolls BJ. Serving a variety of vegetables and fruit as a snack increased intake in preschool children. Am J Clin Nutr 2013; 98(3):693699.
16. Longo-Silva G, Toloni MHA, Rodrigues S, Rocha A, Taddei JAAC. Qualitative evaluation of the menu and plate waste in public day care centers in São Paulo city, Brazil. Rev Nutr 2013; 26(2):135-144.

17. Blondin SA, Djang HC, Metayer N, Anzman-Frasca $S$, Economos CD. 'It's just so much waste.' A qualitative investigation of food waste in a universal free School Breakfast Program. Public Health Nutr 2015; 18(9):1565-1577.

18. Centro Colaborador em Alimentação e Nutrição Escolar (CECANE). Manual para aplicação dos testes de aceitabilidade no Programa Nacional de Alimentação Escolar-PNAE. Brasília: CECANE; 2010.

19. Batista Filho M, Batista LV. Transição alimentar/ nutricional ou mutação antropológica? Cienc. Cult. 2010; 62(4):26-30.

20. Freire MCM, Balbo PL, Amador MA, Sardinha LM. Dietary guidelines for the Brazilian population: implications for the Brazilian National Policy on Oral Health. Cad Saude Publica 2012; 28(Supl.):s20-29.

21. Brasil. Ministério da Saúde (MS). Alimentos regionais brasileiros. Brasília: MS; 2015.

22. Chaves LG, Mendes PNR, Brito RR, Botelho RBA. The national school food program as a promoter of regional food habits. Rev Nutr 2009; 22(6):857-866.

23. Brasil. Ministério da Educação (MEC). Fundo Nacional de Desenvolvimento da Educação (FNDE). Nota Técnica $n^{\circ}$ 01/2014. Restrição da oferta de doces e preparações doces na alimentação escolar. Brasília: MEC; 2014.

24. Dickin K, Griffiths M, Piwoz E. Designing by Dialogue. A Program Planner's Guide to Consultative Research for Improving Young Child Feeding Washington. Washington: Support for Analysis and Research in Africa, Academy for Educational Development; 1997.

25. Neelon SEB, Reyes-Morales H, Haines J, Gillman MW, Taveras EM. Nutritional quality of foods and beverages on child-care centre menus in Mexico. Public Health Nutr 2013; 16(11):2014-2022.

26. Lyn R, Maalouf J, Evers S, Davis J, Griffin M. Nutrition and Physical Activity in Child Cara Centers: The impact of a Wellness Policy Initiative on Environment on Policy Assessment and Observation Outcomes, 2011. Prev Chronic Dis 2013; 10:E83.

27. Sweitzer SJ, Briley ME, Robert-Gray C. Do sack lunches provided by parents meet the nutritional needs of young children who attend child care? J Am Diet Assoc 2009; 109(1):141-144.

28. Clark MA, Fox MK. Nutritional quality of the diets of US public school children and the role of the school meal programs. J Am Diet Assoc 2009; 109(Supl. 2):S44-S56.

29. Byker CJ, Farris AR, Marcenelle M, Davis GC, Serrano EL. Food Waste in a School Nutrition Program After Implementation of New Lunch Program Guidelines. J Nutr Educ Behav 2014; 46(5):406-411.

30. Henry C, Whiting SJ, Phillips T, Finch SL, Zello GA, Vatanparast H. Impact of the removal of chocolate milk from school milk programs for children in Saskatoon, Canada. Appl Physiol Nutr Metab 2015; 40(3):245-250. 
31. Frampton AM, Sisson SB, Horm D, Campbell JE, Lora K, Ladner JL. What's for Lunch? An Analysis of Lunch Menus in 83 Urban and Rural Oklahoma Child-care Centers providing all-day care to preschool children. J Acad Nutr Diet 2014; 114(9):1367-1374.

32. Black RE, Victora CG, Walker SP, Bhutta ZA; Christian P, Onis M, Ezzati M, Grantham-McGregor S, Katz J, Martorell R, Uauy R. Maternal and child undernutrition and overweight in low-income and middle-income countries. Lancet 2013; 382(9890):427-451.

33. McAfee AJ, McSorley EM, Cuskelly GJ, Moss BW, Wallace JM, Bonham MP, Fearon AM. Red meat consumption: an overview of the risks and benefits. Meat Sci 2010; 84(1):1-13.

34. Ferreira HS, Moura RMM, Assunção ML, Horta BL. Fatores associados à hipovitaminose $\mathrm{A}$ em crianças menores de cinco anos. Rev Bras Saude Mater 2013; 13(3):223-235.

35. Brasil. Ministério da Saúde (MS). Pesquisa Nacional de Demografia e Saúde da Criança e da Mulher - PNDS, 2006. Dimensões do processo reprodutivo e saúde da criança. Brasília: MS; 2009.

36. Wessells KR, Brown KH. Estimating the global prevalence of zinc deficiency: results based on zinc availability in national food supplies and the prevalence of stunting. PloS One 2012; 7(11):e50568.

37. Brown KH, Rivera JA, Bhutta Z, Gibson RS, King JC, Lönnerdal B, Ruel MT, Sandtröm B, Wasantwisut E, Hotz C. International Zinc Nutrition Consultative Group (IZiNCG) technical document \#1. Assessment of the risk of zinc deficiency in populations and options for its control. Food Nutr Bull 2004; 25(1 Suppl. 2):S99-203.

38. Prasad AS. Discovery of human zinc deficiency: 50 years later. J Trace Elem Med Biol 2012; 26(2-3):66-69.

39. Fundo Nacional de Desenvolvimento da Educação (FNDE). Ministério da Educação, 2015. [acessado 2016 Out 26]. Disponível em: http://www.fnde.gov.br/ programas/alimentacao-escolar/alimentacao-escolar -apresentacao

40. Chaves LG, Santana TCM, Gabriel CG, Vasconcelos FAG. Reflections on the activities of nutritionists on the Brazilian School Nutrition Program. Cien Saude Colet 2013;18(4):917-926.

41. Fundo Nacional de Desenvolvimento da Educação (FNDE). Alunado por ação do programa nacional de alimentação escolar. 2014. [acessado 2016 Out 28]. Disponível em: http://www.fnde.gov.br/pnaeweb/publico/ relatorioDelegacaoEstadual

Artigo apresentado em 25/08/2016

Aprovado em 03/02/2017

Versão final apresentada em 05/02/2017 